\title{
SOLVATION OF PHOTOLYTICALLY GENERATED p-AMINOPHENYLTHIYL RADICALS STUDIED BY SUB-PICOSECOND TRANSIENT ABSORPTION
}

\author{
Niko P. ERNSTING \\ Max-Planck-Institut für Biophysikalische Chemie, Abteilung Laserphysik, \\ Postfach 2481, D-3400 Göttingen. Federal Republic of Germany
}

Received 21 November 1989; in final form 11 December 1989

\begin{abstract}
By pump-and-probe spectroscopy, the photolysis of bis-( $p$-aminophenyl) disulfide in diethyl ether has been followed with subpicosecond time resolution. The visible absorption band of generated $p$-aminophenylthiyl radicals rises in two stages: an unresolved fast initial rise is followed by a second, exponential rise with a time constant of $5.9 \mathrm{ps}$. During the second process, the peak absorption of the band shifts from its initial position at 557.9 to $572.0 \mathrm{~nm}$. A rate-equation model quantitatively accounts for the observations. The first stage is assigned to the formation of the radical pair in a common solvent cage, and the second stage to the separation and solvation of the radicals.
\end{abstract}

\section{Introduction}

Aromatic disulfides display one major absorption band in the region $240-250 \mathrm{~nm}$, and one or more shoulders may be observed in the region $260-310 \mathrm{~nm}$ [1]. For example, fig. 1 shows the chemical structure and the absorption spectrum of bis-( $p$-aminophenyl) disulfide (I). Flash photolysis of this and other aromatic sulfur molecules results in transient

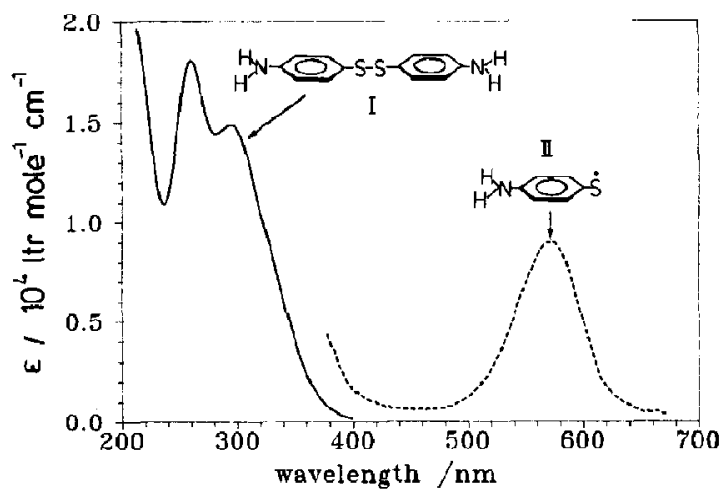

Fig. 1. Absorption spectrum of bis-( $p$-aminophenyl) disulfide (I) and of the $p$-aminophenylthiyl radical (II) in diethyl ether. The radical absorption spectrum was recorded 6.83 ps after photolysis, and it was scaled to a peak extinction coefficient $\epsilon_{\max }=9077$ $\ell \mathrm{mol}^{-1} \mathrm{~cm}^{-1}$ taken from ref. [7]. absorption in the visible spectral range which could be attributed to the corresponding phenylsulfur radical [2]. In comparison with the unsubstituted phenylthiyl radical, the $p$-aminophenylthiyl radical (structure II in fig. 1) has a relatively intense and sharp absorption band which is located at longer wavelengths (dashed line in fig. 1) [2]. Furthermore, solvent shifts of its absorption and emission bands indicate a ground-state dipole moment of 4.3 $D$, which increases upon electronic excitation to 7.3 D [3]. Both findings were interpreted as evidence for the participation of a bipolar, charge-transfer resonance structure which involves the electron accepting properties of the vacant $d$ orbitals of sulfur [3]. Increasing solvent polarity was also shown to reduce the rate of addition of the p-aminophenylthiyl radical to styrene [4].

Laser emission has been obtained from excited $p$ aminophenylthiyl, however only in solution of diethyl ether, tetrahydrofuran, and p-dioxane [5].

To summarize: spectra, reactivity, and lasing efficiency of the $p$-aminophenylthiyl radical depend sensitively on solvent properties. Therefore, this radical should be a convenient probe for microscopic solvent dynamics following photolysis of the parent compound.

This approach was used in ref. [6] (and earlier 
work cited there) by studying the nascent radical absorption, with a time resolution of $\approx 8 \mathrm{ps}$. No spectral evolution was observed for solutions in hexane or in diethylether. In polar solvents, a second, redshifted absorption band was observed in the transient spectra which was attributed to a distinct bipolar form of the radical in the ground state. In ethanol, the second absorption band was seen to rise with a time constant of $25 \mathrm{ps}$, which in this context should correspond to the solvent relaxation time.

Here we report measurements of transient absorption by nascent $p$-aminophenylthiyl radicals with subpicosecond time resolution. Two radicals are generated in close proximity by photolysis of bis- $(p-$ aminophenyl) disulfide in diethyl ether. The evolution of the radical absorption band - i.e. of peak absorbance and spectral position - is well described by simple rate equations.

\section{Experimental}

Bis-( $p$-aminophenyl) disulfide ${ }^{\# 1}$ had been recrystallized several times from ethanol. The solvent was diethyl ether (Merck Uvasol); the disulfide concentration was $4.91 \times 10^{-4} \mathrm{~mol} / \ell$ corresponding to a molecular number density of $2.96 \times 10^{17} \mathrm{~cm}^{-3}$.

The spectrometer for transient absorption is described in ref. [8]. It uses a colliding-pulse-mode dye laser in combination with a $\mathrm{XeCl}$ excimer laser. The pump pulse at $308 \mathrm{~nm}$ had an energy density of $\approx 8$ $\mathrm{mJ} / \mathrm{cm}^{2}$ at the sample, the pulse duration was 0.26 ps. Optical path length was $0.14 \mathrm{~cm}$, and the optical density for pump light was 0.92 .

The probe pulse consisted of a white light continuum generated in $o$-phosphoric acid and silica glass. It was polarized at the magic angle relative to the polarization of the pump light. Transient absorption was measured by recording sample and reference spectra simultaneously. The overall time resolution may be given by the spectral intensity cross correlation between the pump and probe pulse: its fwhm was 0.35 ps. For a determination of time zero for probe wavelengths around $600 \mathrm{~nm}$, ground-state

\#I A gift from Dr. Shin-ichi Ishizaka. bleaching of malachite green in methanol was used [8].

\section{Results}

The visible absorption band of the $p$-aminophenylthiyl radical in diethyl ether has its peak near 570 nm [3]. Fig. 2 shows the evolution of transient absorption toward this band, after photolysis of bis- $(p$ aminophenyl) disulfide at $308 \mathrm{~nm}$ with an intense pulse of $0.26 \mathrm{ps}$ duration. At the earliest time shown here, i.e. at $0.17 \mathrm{ps}$, there is a background of broad absorption throughout the visible range which is superimposed by an incipient band maximizing at 558 $\mathrm{nm}$. The rise of this band follows the integrated pump pulse. After the photolysis pulse has passed, at times later than $\approx 0.4 \mathrm{ps}$, the characteristic absorption band continues to increase while the band maximum shifts towards longer wavelengths.

The transient absorption spectrum recorded at 6.83 ps is again shown in fig. 3 (circles). The radical absorption band can be well described by a sum of two Gaussian functions (on the energy scale) and a constant background. The best fit is shown as solid line in the figure. Low-temperature studies [9] suggest that this description is merely convenient and should not be interpreted in terms of vibronic structure.

For every transient spectrum, the radical absorption band was fitted in this way in order to accurately determine its absorption maximum. The max-

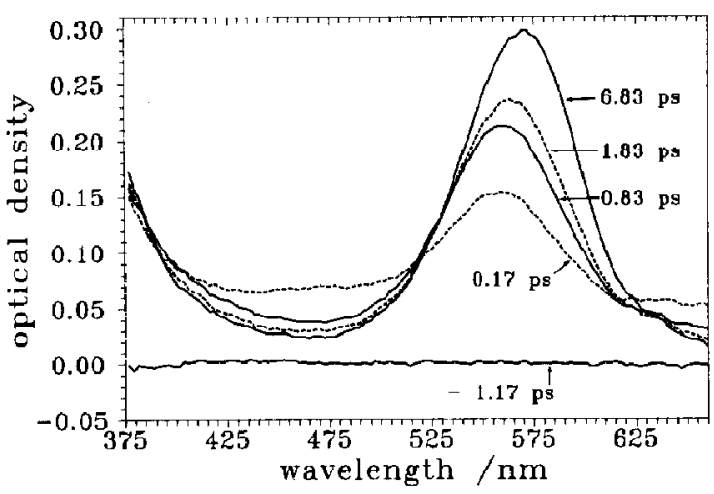

Fig. 2. Transient absorption spectra in the course of photolysis of bis-( $p$-aminophenyl) disulfide in diethyl ether. The original data points have been connected by straight lines. 


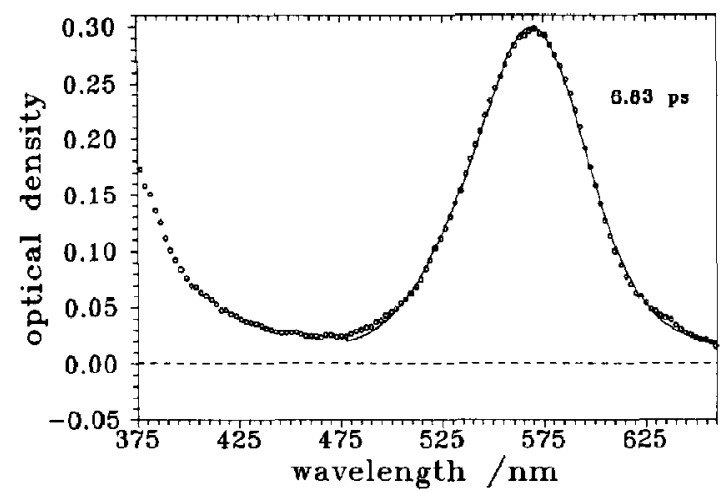

Fig. 3. Absorption spectrum (circles) due to p-aminophenylthiyl radicals, measured $6.83 \mathrm{ps}$ after photolytic generation with an intense pump pulse at $308 \mathrm{~nm}$ with 0.26 ps duration (fwhm). The solid line shows the best fit by two Gaussian functions (on the energy scale) and a spectrally constant background.

imum value of optical density could be determined with a precision of \pm 0.002 , and the relative spectral position of the absorption maximum could be located within $\pm 10 \mathrm{~cm}^{-1}$. In particular, the incipient band seen at $0.17 \mathrm{ps}$ after photolysis (cf. fig. 2) has its maximum at $557.9 \mathrm{~nm}$.

The peak transient optical density, as function of time, is shown as solid circles in fig. $4 a$. The dashed line indicates the calculated hypothetical behaviour of peak optical density in case that photodissociation occurs instantaneously. Fig. $4 \mathrm{~b}$ shows the same results on an expanded time scale. Fig. $4 c$ shows the temporal evolution of the spectral position for the absorption maximum.

\section{Discussion}

The results of fig. 4 suggest that the photodissociation of bis- ( $p$-aminophenyl) disulfide in diethyl ether to form two $p$-aminophenylthiyl radicals proceeds in two stages: first, instantaneous generation of roughly half of the final radical absorption; second, further increase of absorption with an approximate time coefficient of 7 ps (see below). The latter process is accompanied by a continuous bathochromic shift of the absorption maximum, from 557.9 to $572.0 \mathrm{~nm}$.

What can be the underlying physical reason for the observed rise of radical absorption after the photol-
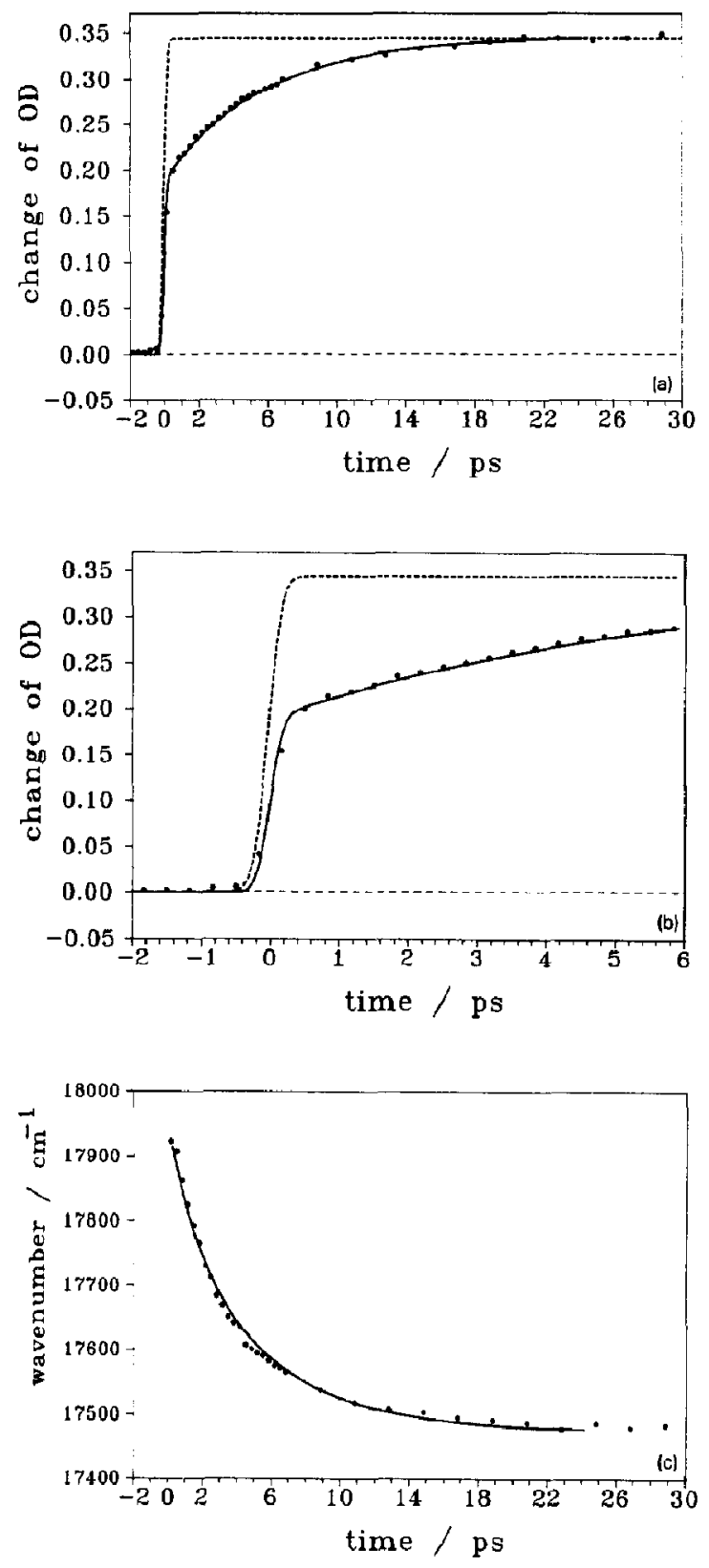

Fig. 4. Temporal evolution of optical density at the peak of the radical absorption band at $\approx 560 \mathrm{~nm}$ (circles). The dashed line represents the integrated apparatus function. The solid line gives the best fit obtained from a ratc-cquation model. (b) Temporal evolution of optical density at the peak of the radical absorption band, as in (a), but on an expanded time scale. (c) Spectral position of the peak radical absorption, as a function of time (circles). The solid line gives the best fit obtained from a rate-equation model. 
ysis pulse is over? Orientational diffusion may be excluded because the measurements were performed at the magic angle between the pump and probe polarizations. Cooling of radicals which are initially hot may also be ruled out for the following reason. The S-S bond dissociation energy of diphenyl disulfide is $2.8 \mathrm{eV} \mathrm{[10],} \mathrm{while} \mathrm{the} \mathrm{photon} \mathrm{energy} \mathrm{of} \mathrm{excitation}$ (at $308 \mathrm{~nm}$ ) is $4.03 \mathrm{eV}$. Hence immediately after photodissociation, each radical would remain with $0.62 \mathrm{eV}$, or $5000 \mathrm{~cm}^{-1}$, of excess vibrational energy. It may be estimated that this excess energy would result in a molecule which is heated from room temperature to less than $500 \mathrm{~K}$. Subsequent energy dissipation to the surrounding medium may be responsible for a gradual steepening of the red edge of the absorption band, as is clearly seen in fig. 2 . However, cooling cannot account for the observed increase of the integrated absorbance.

Instead, the time scale of tens of picoseconds and the accompanying spectral shifts suggest solvation as the main mechanism behind the spectral evolution. The increase in peak optical density may be viewed rather as reduced absorption for the radicals when they are still in close proximity, e.g. within the solvent cage inherited from the parent compound. The relatively small absorption by these radicals can be due to two reasons: a reduced transition oscillator strength per radical, or a reduced number of radicals present at an early time because of an ultrafast "equilibrium" (to be specified below) with the excited parent compound.

Let us first consider the case of reduced oscillator strength. The geminate radicals will certainly interact by the dipole-dipole mechanism, and possibly by the electron exchange mechanism [11]. Both result in the formation of exciton states; the corresponding optical transition has modified oscillator strength and an altered vibronic structure compared to that of the solvated radical. This hypothetical situation is similar to that of anthracene dimers generated by photolysis of dianthracene in a rigid matrix at low temperatures [12-14]; it is depicted in fig. 5a.

The second case - ultrafast "equilibrium" with the excited parent - is shown schematically in fig. 5 b. Here it is assumed that the dissociation is impeded by the solvent cage, and that the radical pair can recombine in the course of librational motion to form the excited parent molecule. Optical transitions in
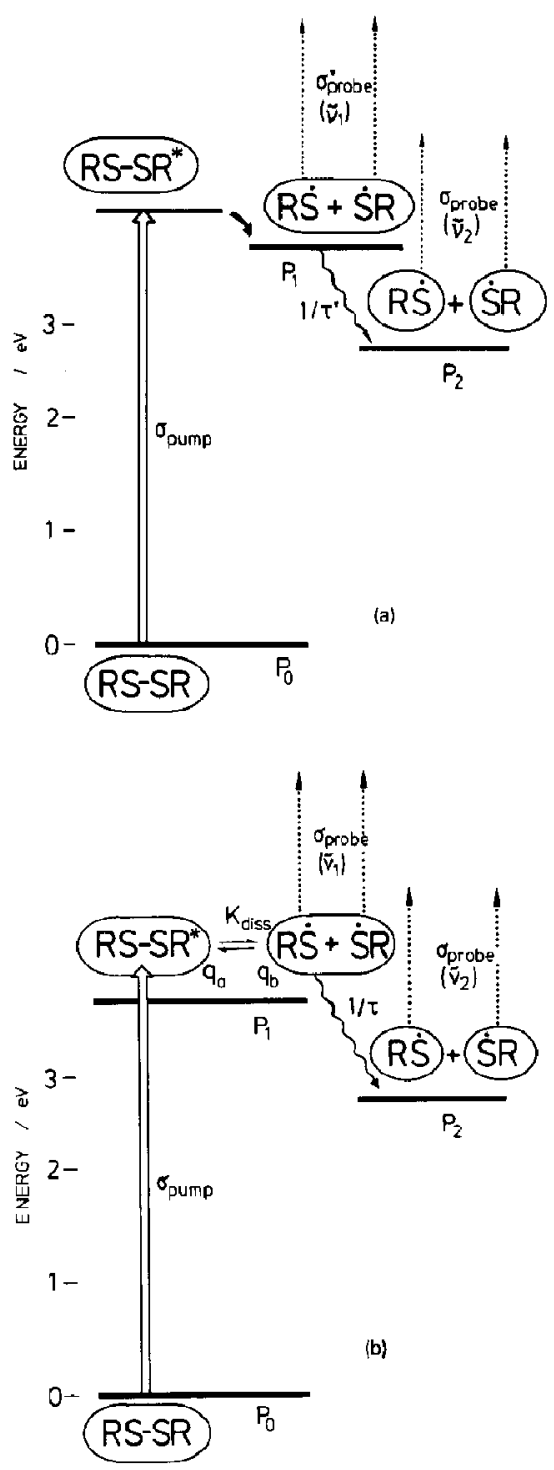

Fig. 5. Two models for the photolysis of bis-( $p$-aminophenyl $)$ disulfide (=RSSR) into $p$-aminophenylthiyl radicals (= RS ). Large circles indicate a solvent cage. (a) Ultrafast generation of the radical pair, with reduced absorption cross section $\sigma_{\text {probs; }}^{\prime}$ (b) librational "equilibrium" between the excited parent molecule and the radical pair.

the visible range would come from that part of the librational probability density function which is close to the classical turning point at largest distance within the cage. (For a review of cage effects see ref. [15].)

The two models differ in prediction for the temporal evolution of parent ground-state bleaching. For 
the simulation of the data presented here, both models are equivalent. Therefore we discuss in the following mainly the "equilibrium" model shown in fig. 5 b.

Let $P_{0}$ and $P_{1}$ denote the probability that the disulfide parent molecule is in the electronic ground or in the excited state, respectively, within the original solvent cage, while $\boldsymbol{P}_{2}$ gives the probability that the molecule is separated into two solvated radicals. For the situation within the original solvent cage, $q_{\mathrm{a}}$ and $q_{\mathrm{b}}$ represent the probability of finding the excited parent molecule or the radical pair. With

$P_{0}=1-P_{1}-P_{2}$,

$P_{\mathrm{l}}=q_{\mathrm{a}}+q_{\mathrm{b}}$,

$q_{\mathrm{b}}=K_{\text {diss }} q_{\mathrm{a}}$,

we then have the rate equations

$\dot{P}_{1}=I(t) \sigma_{\text {pump }} P_{0}-K_{\text {diss }}\left(1+K_{\text {diss }}\right)^{-1} \tau^{-1} P_{1}$,

$\dot{P}_{2}=+K_{\text {diss }}\left(1+K_{\text {diss }}\right)^{-1} \tau^{-1} P_{1}$.

(Here, $I(t)$ is the pump photon flux: we assume a Gaussian pulse normalized to the pump fluence. $\sigma_{\text {pump }}$ is the absorption cross section of the disulfide compound at $308 \mathrm{~nm}$, and $\tau$ represents the intrinsic lifetime of the radical pair.) The first observable is the optical density at the peak of the radical absorption band:

$\mathrm{OD}_{\text {probe }}=\log _{10}(e) N d \times 2 \sigma_{\text {probc }}\left(q_{\mathrm{b}}+P_{2}\right)$.

( $N$ is the original number density of the parent molecule, $d$ the optical path length, and $\sigma_{\text {probe }}$ the peak absorption cross section for one radical.)

The second observable is the wavenumber $\bar{\nu}$ for the peak of the radical absorption band. For simplicity, we approximate $\tilde{\nu}$ by the weighted average of wavenumber $\tilde{\nu}_{1}$ for a radical in the cage, and $\tilde{\nu}_{2}$ for a separately solvated radical:

$\tilde{\nu}=\left(q_{\mathrm{b}} \tilde{\nu}_{1}+P_{2} \tilde{\nu}_{2}\right) /\left(q_{\mathrm{b}}+P_{2}\right)$.

Eqs. (2) and (3) were integrated; the calculated curves $\operatorname{OD}_{\text {probe }}(t)$ and $\tilde{\nu}(t)$ were then convoluted with a normalized Gaussian function representing the probe pulse. The best fit to the measured data is shown as solid lines in fig. 4 . It can be seen that the temporal evolution is completely reproduced to within the uncertainty of the measurements. The parameters which were used in the fit are collected in table 1 . In particular, the decay of the probability $q_{\mathrm{b}}$ for the radical pair in the original solvent cage has an intrinsic time constant $\tau=3.2 \mathrm{ps}$. However, $q_{\mathrm{b}}$ is

Table 1

Parameters used for the rate-equation model

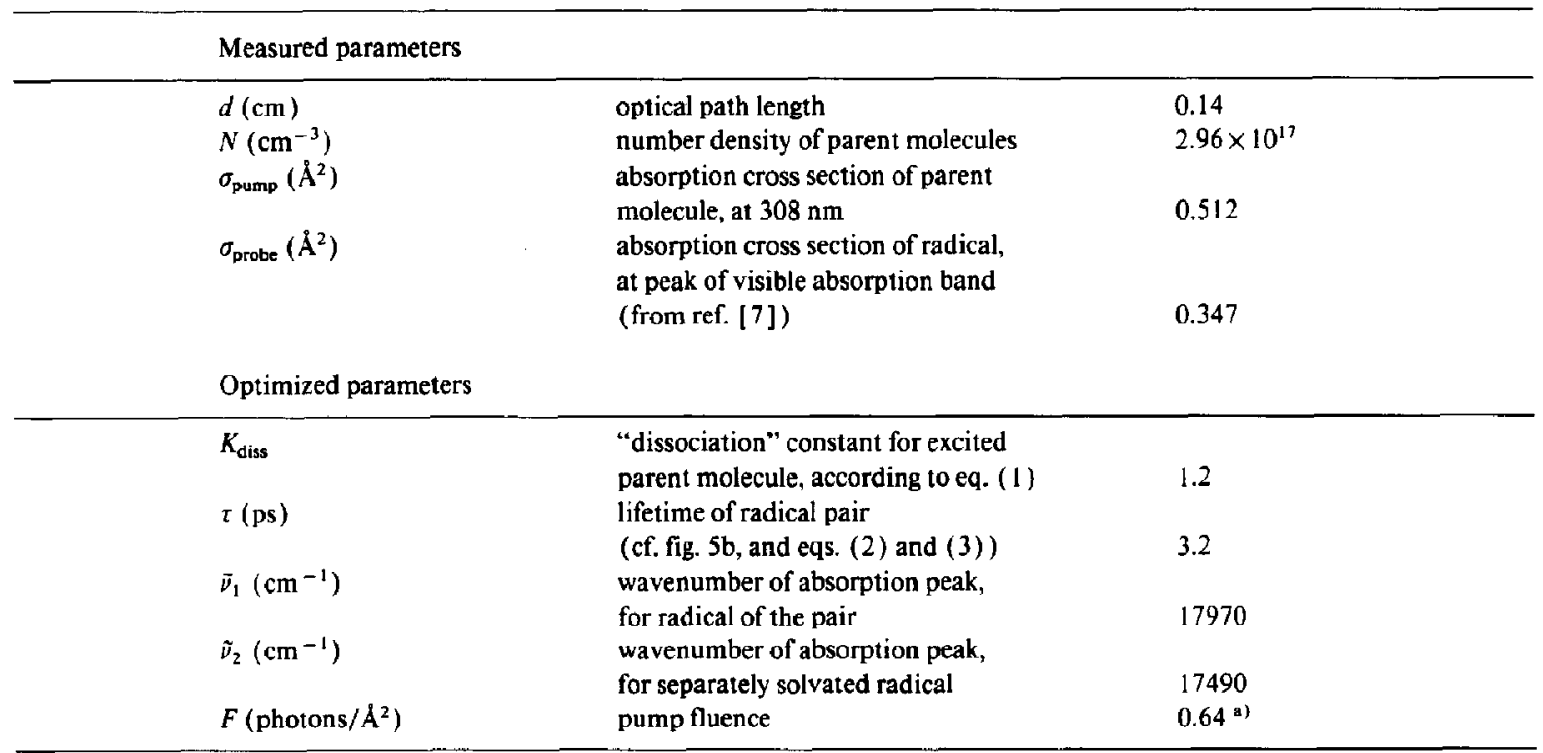

a) This value is about half of the one determined from the pulse energy and an estimate of the illuminated area. 
fed through the librational "equilibrium". This results in a kinetic curve for the observable rise of radical absorption which is exponential, with time constant $\tau\left(1+K_{\text {diss }}\right) / K_{\text {diss }}=5.9$ ps.

Finally, consider the first model mentioned above. In this context, the parameters mentioned so far may simply be reinterpreted. The decay of the radical pair is given by the decay of the probability $P_{1}$. The corresponding time constant is $\tau^{\prime}=\tau\left(1+K_{\text {diss }}\right) / K_{\text {diss }}$ $=5.9 \mathrm{ps}$. The probe absorption cross section for one radical in the pair, $\sigma_{\text {probe }}^{\prime}$, is reduced from that of the separately solvated radical: $\sigma_{\text {probe }}^{\prime}=K_{\text {diss }}(1+$ $\left.K_{\text {diss }}\right)^{-1} \sigma_{\text {probe }}=0.55 \sigma_{\text {probe- }}$

\section{Conclusion}

The photolysis of bis-( $p$-aminophenyl) disulfide in diethyl ether was followed by monitoring the welldefined visible absorption band of the generated $p$ aminophenylthiyl radicals. We observe an instantaneous rise which is followed by an exponential rise with a time constant of $5.9 \mathrm{ps}$; the latter rise is associated with a continuous red-shift of the absorption maximum. Thus the reaction proceeds in two stages.

A rate equation model quantitatively accounts for the observations. The first stage is assigned to the formation of the radical pair in a common solvent cage. The corresponding absorption band has its maximum at $557.9 \mathrm{~nm}$, and the effective absorption at that wavelength per radical is reduced from that of the separately solvated radicals. The second stage is assigned to solvation of the radicals.

\section{Acknowledgement}

The author is greatly indebted to Dr. S. Ishizaka for introduction into this topic. Stimulating discussions with Professor M. Kotani, Dr. K.H. Grellmann, and Dr. R. Schmidt are gratefully acknowledged, as is support by Professor F.P. Schäfer and the Deutsche Forschungsgemeinschaft through the Leibniz Prize program.

\section{References}

[1] W.W. Simons, ed., The Sadtler handbook of ultraviolet spectra (Heyden, London, 1979) pp. 357-359.

[2] F.C. Thyrion, J. Phys. Chem. 77 (1973) 1478.

[3] G.H. Morine and R.R. Kuntz, Chem. Phys. Letters 67 (1979) 552.

[4] O. Ito and M. Matsuda, J. Am. Chem. Soc. 104 (1982) 568.

[5] S. Ishizaka and M. Kotani, Chem. Phys. Letters 117 (1985) 251.

[6] N.A. Lysak, S.V. Mel'nichuk, S.A. Tikhomirov and G.B. Tolstorozhev, Zh. Prikl. Spektrosk. 49 (1988) 949.

[7] N.A. Borisevich, N.A. Lysak, S.V. Mel'nichuk, S.A. Tikhomirov and G.B. Tolstorozhev, Lecture Sa2 of the 6th International Symposium on Ultrafast Phenomena in Spectroscopy, Neubrandenburg, GDR (Aug. 23-27, 1989).

[8] N.P. Ernsting and M. Kaschke, Appl. Phys. B, submitted for publication.

[9] S. Ishizaka, unpublished results.

[10] H. Mackle and R,G. Mayrick, Trans. Faraday Soc. 58 (1962) 238.

[11] D.L. Dexter, J. Chem. Phys. 21 (1953) 836.

[12] E.A. Chandross, J. Chem. Phys. 43 (1965) 4175.

[13] J. Ferguson, A.W.-H. Mau and J.M. Morris, Australian J. Chem. 26 (1973) 91.

[14] J. Ferguson, A.W.-H. Mau and J.M. Morris, Australian J. Chem. 26 (1973) 103.

[15] J. Troe, J. Phys. Chem. 90 (1986) 357. 